\title{
Adaptive strategies for system of fuzzy differential equation: application of arms race model
}

\author{
Sankar Prasad Mondal ${ }^{a}$, Najeeb Alam Khan ${ }^{b, *}$, Oyoon Abdul Razzaq ${ }^{c}$, Suklal Tudu ${ }^{d}$, Tapan Kumar Roy ${ }^{d}$ \\ ${ }^{a}$ Department of Mathematics, Midnapore College (Autonomous), Midnapore, West Midnapore-721101, West Bengal, India. \\ ${ }^{b}$ Department of Mathematics, University of Karachi, Karachi 75270, Pakistan. \\ ${ }^{c}$ Department of Humanities and Natural Sciences, Bahria University, Karachi 75260, Pakistan. \\ ${ }^{d}$ Department of Mathematics, Indian Institute of Engineering Science and Technology, Shibpur, Howrah-03, West Bengal, India.
}

\begin{abstract}
The paper presents adaptive stratagems to scrutinize the system of first order fuzzy differential equations (SFDE) in two modes, fuzzy and in crisp sense. Its fuzzy solutions are carried out using two approaches, namely, Zadeh's extension principle and generalized Hukuhara derivative (gH-derivative). While, different defuzzification techniques; central of area method (COA), bisector of area method (BOA), largest of maxima (LOM), smallest of maxima (SOM), mean of maxima (MOM), regular weighted point method (RWPM), graded mean integration value (GMIV), and center of approximated interval (COAI), are employed to discuss the crisp solutions. Moreover, the arms race model (ARM), which have a significant implication in international military planning, are pragmatic examples of system of first order differential equations, but not studied in fuzzy sense, hitherto. Therefore, ARM is re-established and studied here with fuzzy numbers to estimate its uncertain parameters, as a practical utilization of SFDE. Additionally, an illustrative example of ARM is undertaken to clarify the appropriateness of the proposed approaches.
\end{abstract}

Keywords: Fuzzy differential equation, defuzzification, Hukuhara derivative, extension principle.

2010 MSC: 34A07.

(C) 2018 All rights reserved.

\section{Introduction}

After the introduction and major innovations in the theory of fuzzy differential equations $[4,6,14]$, the term fuzzy differential equation (FDE) is instantaneously growing as a new area in fuzzy calculus. These equations are acquired interchangeably by incorporating differential equations with fuzzy initial values, fuzzy boundary values or with fuzzy functions as well. Pertinently applicable to reformulate many dynamic models with multiple uncertain parameters, FDEs have noteworthy apperception in various fields of biology, engineering, physics, and so forth. In this regard, up till now, many attempts have been made in developing different approaches to discuss the solutions of FDEs [1, 16, 17, 20].

Moreover, SFDEs are considered to be prominently in the lead among all the FDEs. These equations have wide applications in different aspects to handle uncertainty of numerous real-world phenomenon.

\footnotetext{
${ }^{*}$ Corresponding author

Email address: njbalam@yahoo.com (Najeeb Alam Khan)

doi: $10.22436 /$ jmcs.018.02.07
} 
For example, population dynamics model [2], modeling hydraulic [5], bioinformatics and computational biology [7], quantum optics and gravity [10], HIV model [24], friction model [3], and decay model [9] to name a few. Consequently, many techniques have been employed to investigate the solutions of SFDEs, for instance, Gasilova et al. [12] described system of differential equations with fuzzy initial conditions and applied a geometric approach to obtain its solutions as a fuzzy set of vector functions, Fard et al. [11] discussed nonhomogeneous $n$-dimensional system of fuzzy differential equations and obtained its approximate-analytical solutions using variational iteration method etc. [15, 25].

In spite of above mentioned developments, lacunas still exist in the formulation and solution of many realistic models of complementary items. In this connection, here arms race model has been restructured and deliberated in fuzzy environment to undergo these lacunas. ARMs, also known as Richardson's model [13, 18], have been intensively constructed by many political scholars. It is mainly studied to specify the structural and behavioral properties of the international system so that the military of the respective country can have complete hegemony over the opponent during a war. The novelty in present endeavor is that the uncertain parameters of ARM is also taken into consideration by using fuzzy numbers. Two approaches, Zadeh's extension principle [19] and gH-derivative [1, 4], are conducted to illustrate the fuzzy solutions. Furthermore, crisp solutions are also calculated by defuzzifying the fuzzy solutions using different defuzzification methods [8, 21-23].

\section{Preliminaries}

Bearing in mind the prerequisites of the paper, in this section, we give some necessary descriptions and notions related to fuzzy set theory and fuzzy calculus, briefly.

\subsection{Fuzzy set theory}

Let $A_{F}$ be a set on real line $\mathfrak{R}$ defined as $A_{F}=\{\tau \mid \tau: \mathfrak{R} \longrightarrow[0,1]\}$, such that $\tau$ is normal, fuzzy convex, upper semi continuous and compactly supported on $\Re$. Then $\tau$ is a fuzzy number and $A_{F}$ is said to be the set of fuzzy numbers. Each $\tau$ may be expressed as nonempty compact intervals, $[\tau]^{\alpha}=\left[\tau_{1}(\alpha), \tau_{2}(\alpha)\right]$ for all $\alpha \in[0,1]$, which are said to be $\alpha$-level sets or $\alpha$-cuts of $\tau$. The terms $\tau_{1}(\alpha)$ and $\tau_{2}(\alpha)$ represent its non-decreasing lower and non-increasing upper functions, accordingly, with $\tau_{1}(\alpha) \leqslant \tau_{2}(\alpha)$ and both are bounded left continuous on $(0,1]$ and right continuous at $\alpha=0$.

Different types of fuzzy numbers exist in literature with respect to their membership functions, but in this sequel, we use the triangular fuzzy numbers. Thus, a triangular fuzzy number $\Lambda$ is represented with the help of vertices of triangle, i.e., $\left(a_{1}, a_{2}, a_{3}\right)$, where $a_{1}<a_{2}<a_{3}$ such that $\left(a_{1}, a_{3}\right)$ measures the base and $a_{2}$ is the height of the triangle. Its membership function is defined as

$$
\Omega_{\wedge}(x)= \begin{cases}\frac{x-a_{1}}{a_{2}-a_{1}}, & \text { if } a_{1} \leqslant x \leqslant a_{2} \\ \frac{a_{3}-x}{a_{3}-a_{2}}, & \text { if } a_{2} \leqslant x \leqslant a_{3} \\ 0, & \text { otherwise }\end{cases}
$$

with its $\alpha$-level set as, $[\Lambda]^{\alpha}=\left[\Lambda_{1}(\alpha), \Lambda_{2}(\alpha)\right]=\left[a_{1}+\left(a_{2}-a_{1}\right) \alpha, a_{3}-\left(a_{3}-a_{2}\right) \alpha\right]$. Moreover, algebraic operations of fuzzy numbers can be defined as, $\forall \mathrm{u}, \nu \in A_{\mathrm{F}}$ and $\forall \alpha \in[0,1]$,

a.

$$
[u \oplus v]^{\alpha}=[u]^{\alpha} \oplus[v]^{\alpha}=\left[u_{1}(\alpha)+v_{1}(\alpha), u_{2}(\alpha)+v_{2}(\alpha)\right]
$$

b. $\forall c \in \Re$

$$
[\mathrm{cu}]^{\alpha}=\mathrm{c}[u]^{\alpha}= \begin{cases}{\left[\mathrm{cu}_{1}(\alpha), \mathrm{cu}_{2}(\alpha)\right],} & \text { if } c>0 \\ \{0\}, & \text { if } c=0 \\ {\left[\mathrm{cu}_{2}(\alpha), \mathrm{cu}_{1}(\alpha)\right],} & \text { if } c<0\end{cases}
$$


c.

$$
\begin{aligned}
& {[u \odot v]^{\alpha}=\left[\min \left\{u_{1}(\alpha) v_{1}(\alpha), u_{1}(\alpha) v_{2}(\alpha), u_{2}(\alpha) v_{1}(\alpha), u_{2}(\alpha) v_{2}(\alpha)\right\}\right.} \\
& \left., \max \left\{u_{1}(\alpha) v_{1}(\alpha), u_{1}(\alpha) v_{2}(\alpha), u_{2}(\alpha) v_{1}(\alpha), u_{2}(\alpha) v_{2}(\alpha)\right\}\right],
\end{aligned}
$$

where $\oplus$ and $\odot$ define fuzzy addition and fuzzy multiplication, respectively. Whereas, the quantity,

$$
D_{\mathrm{F}}(u, v)=\left(\int_{0}^{1}\left(u_{1}-v_{1}\right)^{2}+\int_{0}^{1}\left(u_{2}-v_{2}\right)^{2}\right)^{\frac{1}{2}}
$$

defines the distance between fuzzy numbers $u$ and $v[4,16]$.

\subsection{Generalized Hukuhara Difference}

For the fuzzy numbers $u$ and $v$, the gH-difference is defined as

$$
\Omega_{\wedge}(x)= \begin{cases}\frac{x-a_{1}}{a_{2}-a_{1}}, & \text { if } a_{1} \leqslant x \leqslant a_{2}, \\ \frac{a_{3}-x}{a_{3}-a_{2}}, & \text { if } a_{2} \leqslant x \leqslant a_{3}, \\ 0, & \text { otherwise, }\end{cases}
$$

and in terms of $\alpha$-level set it is elaborated as, $\forall \alpha \in[0,1]$

$$
u(\alpha) \ominus_{g} H v(\alpha)=\left[\min \left\{u_{1}(\alpha)-v_{1}(\alpha), u_{2}(\alpha)-v_{2}(\alpha)\right\}, \max \left\{u_{1}(\alpha)-v_{1}(\alpha), u_{2}(\alpha)-v_{2}(\alpha)\right\}\right] .
$$

Furthermore, a fuzzy-valued function $\tilde{f}(t)$ is defined as $\tilde{f}: \mathfrak{R} \longrightarrow A_{F}$, with its $\alpha$-level sets, $f(t ; \alpha)=$ $\left[\mathrm{f}_{1}(\mathrm{t} ; \alpha), \mathrm{f}_{2}(\mathrm{t} ; \alpha)\right], \forall \mathrm{t} \in \mathfrak{R}$ and $\forall \alpha \in[0,1]$.

\subsection{Generalized Hukuhara differentiability}

The ground-breaking theory of gH-differentiability of fuzzy-valued functions is a tremendous innovation of Bede et al. [4]. This great contribution in theory of fuzzy calculus has been widely studied and utilized by many inspired researches of this field [1]. Along these lines, the gH-differentiability of fuzzyvalued function $\tilde{f}(t)$ is defined as below: A fuzzy-valued function $\tilde{f}:(a, b) \longrightarrow A_{F}$ is gH-differentiable at $t_{0} \in(a, b)$ if $\tilde{f}^{\prime}\left(t_{0}\right) \in A_{F}$, such that

$$
\widetilde{f}^{\prime}\left(t_{0}\right)=\lim _{h \longrightarrow 0} \frac{\tilde{f}\left(t_{0}+h\right) \ominus_{g H} \tilde{f}\left(t_{0}\right)}{h} .
$$

In $\alpha$-level set it is explained as,

$$
\widetilde{f}^{\prime}(t ; \alpha)=\left[\min \left\{f_{1}^{\prime}(t ; \alpha), f_{2}^{\prime}(t ; \alpha)\right\}, \max \left\{f_{1}^{\prime}(t ; \alpha), f_{2}^{\prime}(t ; \alpha)\right\}\right] .
$$

For the condition that $f_{1}(t ; \alpha)$ and $f_{2}(t ; \alpha)$ are differentiable. In addition, $\tilde{f}(t)$ is said to be (i)-gH differentiable at $t_{0}$ if,

$$
f^{\prime}(t ; \alpha)=\left[f_{1}^{\prime}(t ; \alpha), f_{2}^{\prime}(t ; \alpha)\right]
$$

and $\tilde{f}(t)$ is (ii)-gH differentiable at $t_{0}$ if,

$$
f^{\prime}(t ; \alpha)=\left[f_{2}^{\prime}(t ; \alpha), f_{1}^{\prime}(t ; \alpha)\right] .
$$




\section{System of linear fuzzy differential equations (SLFDE)}

Let $a_{i j}, \widetilde{f}_{i}(t)$, and $\widetilde{g}_{i}$ be crisp numbers, fuzzy functions, and fuzzy numbers, respectively, for $0 \leqslant \widetilde{g}_{i} \leqslant$ $1,1 \leqslant i \leqslant n$, and $j \leqslant n$, then system of fuzzy differential equation is outlined as

$$
\begin{aligned}
\frac{d x_{1}(t)}{d t} & =a_{11} x_{1}(t)+\cdots+a_{1 n} x_{n}(t)+\tilde{f}_{1}(t), \\
\frac{d x_{2}(t)}{d t} & =a_{21} x_{1}(t)+\cdots+a_{2 n} x_{n}(t)+\widetilde{f}_{2}(t), \\
& \vdots \\
\frac{d x_{n}(t)}{d t} & =a_{n 1} x_{1}(t)+\cdots+a_{n n} x_{n}(t)+\widetilde{f}_{n}(t),
\end{aligned}
$$

with fuzzy initial conditions

$$
x_{1}(t)=\widetilde{g}_{1}, x_{2}(t)=\widetilde{g}_{2}, \cdots, x_{n}(t)=\widetilde{g}_{n} .
$$

System (3.1)-(3.2) can also be written in matrix form as

$$
\frac{d X(t)}{d t}=A X(t)+\widetilde{F}(t), \quad X\left(t_{0}\right)=\widetilde{G},
$$

where $A=\left[a_{i j}\right]$ is a $n \times n$ crisp matrix, $\widetilde{F}(t)=\left(\widetilde{f}_{1}(t), \widetilde{f}_{2}(t), \ldots, \widetilde{f}_{n}(t)\right)^{\top}$ is a vector of fuzzy function, and $\widetilde{\mathrm{G}}=\left(\widetilde{\mathrm{g}}_{1}, \widetilde{\mathrm{g}}_{2}, \ldots, \widetilde{\mathrm{g}}_{\mathrm{n}}\right)^{\top}$ is a vector of fuzzy numbers.

\subsection{Zadeh's extension principle}

Zadeh's extension principle plays a vital role in fuzzy calculus, according to this principle, any crisp function can take fuzzy sets as arguments. This principle can be stated as the following.

If a function $\phi: \mathfrak{R}^{\mathrm{m}} \longrightarrow \mathfrak{R}^{\mathfrak{n}}$ induces to another function $\psi: \phi(\mathrm{K}) \longrightarrow \phi(\mathrm{M})$ defined for each fuzzy set $K$ in $\mathfrak{R}^{m}$, we obtain a fuzzy set $M$ in $\mathfrak{R}^{n}$, i.e., let $y \in \mathfrak{R}^{n}$

$$
\phi(M)(y)= \begin{cases}\sup _{x \in f^{-1}(y)} K(x), & \text { if } y \in \operatorname{range}(\phi), \\ 0, & \text { if } y \notin \operatorname{range}(\phi) .\end{cases}
$$

If $\phi$ is one to one mapping, then

$$
\phi(M)(y)= \begin{cases}K\left(f^{-1}(y)\right), & \text { if } y \in \operatorname{range}(\phi), \\ 0, & \text { if } y \notin \operatorname{range}(\phi) .\end{cases}
$$

Rewriting Eq. (3.3) as

$$
X^{\prime}(t)=H((t), x(t)), a \leqslant t \leqslant b, X\left(t_{0}\right)=\widetilde{G},
$$

then, by using the extension principle we have the membership function

$$
\mathrm{Q}(\mathrm{t}, x(\mathrm{t}))(\mathrm{s})=\sup \{x(\mathrm{t})(\sigma) \mid \mathrm{s}=\mathrm{H}(\mathrm{t}, \sigma)\}, \mathrm{s} \in \mathfrak{R} .
$$

The result $Q(t, x(t))(s)$ is a fuzzy function where

$$
\mathrm{Q}_{1}\left(\mathrm{t}, \mathrm{x}_{1}(\mathrm{t} ; \alpha), \mathrm{x}_{2}(\mathrm{t} ; \alpha)\right)(\mathrm{s})=\min \left\{\mathrm{H}(\mathrm{t}, \mathrm{u})(\mathrm{s}) \mid \mathrm{u} \in\left[\mathrm{x}_{1}(\mathrm{t} ; \alpha), \mathrm{x}_{2}(\mathrm{t} ; \alpha)\right]\right\}
$$

and

$$
\mathrm{Q}_{2}\left(\mathrm{t}, \mathrm{x}_{1}(\mathrm{t} ; \alpha), \mathrm{x}_{2}(\mathrm{t} ; \alpha)\right)(\mathrm{s})=\max \left\{\mathrm{H}(\mathrm{t}, \mathrm{u})(\mathrm{s}) \mid \mathrm{u} \in\left[\mathrm{x}_{1}(\mathrm{t} ; \alpha), \mathrm{x}_{2}(\mathrm{t} ; \alpha)\right]\right\}
$$




\subsection{Generalized Hukuhara derivative}

Let $X(t)$ be fuzzy-valued functions in Eq. (3.3), then SLFDE is structured under two different cases.

Case 1: When $X(t)$ is (i)-gH differentiable.

Case 2: When $\mathrm{X}(\mathrm{t})$ is (ii)-gH differentiable.

Illustrating Eq. (3.3) for each case, consecutively, we attain the following SLFDEs.

Case 1: Converting Eq. (3.3) into $\alpha$-level sets of $\mathrm{X}(\mathrm{t})$ under (i)-gH differentiability, we attain two differential equations as

$$
\left.\frac{d X_{1}(t ; \alpha)}{d t}=A X_{1}(t ; \alpha)+F_{1}(t ; \alpha), \quad \frac{d X_{2}(t ; \alpha)}{d t}=A X_{2}(t ; \alpha)+F_{2}(t ; \alpha)\right\}
$$

with initial conditions

$$
X_{1}\left(t_{0} ; \alpha\right)=B_{1}(\alpha), X_{2}\left(t_{0} ; \alpha\right)=B_{2}(\alpha),
$$

where $x_{1}(t ; \alpha)=\left(x_{11}(t ; \alpha), x_{21}(t ; \alpha), \ldots, x_{n 1}(t ; \alpha)\right), x_{2}(t ; \alpha)=\left(x_{12}(t ; \alpha), x_{22}(t ; \alpha), \ldots, x_{n 2}(t ; \alpha)\right)$, $F_{1}(t ; \alpha)=\left(f_{11}(t ; \alpha), f_{21}(t ; \alpha), \ldots, f_{n 1}(t ; \alpha)\right), F_{2}(t ; \alpha)=\left(f_{12}(t ; \alpha), f_{22}(t ; \alpha), \ldots, f_{n 2}(t ; \alpha)\right)$ and $A=$ $\left[a_{i j}\right], a_{i j} \geqslant 0$, is a $n \times n$ crisp matrix.

Case 2: Whereas, under (ii)-gH differentiability of $X(t)$, Eq. (3.3) expands into the following differential equations

$$
\left.\frac{d X_{2}(t ; \alpha)}{d t}=A X_{1}(t ; \alpha)+F_{1}(t ; \alpha), \quad \frac{d X_{1}(t ; \alpha)}{d t}=A X_{2}(t ; \alpha)+F_{2}(t ; \alpha)\right\}
$$

with initial conditions

$$
X_{1}\left(t_{0} ; \alpha\right)=B_{1}(\alpha), X_{2}\left(t_{0} ; \alpha\right)=B_{2}(\alpha),
$$

where $X_{1}(t ; \alpha), X_{2}(t ; \alpha), F_{1}(t ; \alpha), F_{2}(t ; \alpha)$ and $A$ is same as defined in Case 1 .

In this paper, we will only consider two cases

i. All $\widetilde{x}_{i}(t), i=1,2, \ldots, n$ are (i)-gH differentiable and all $a_{i j} \geqslant 0, i, j=1,2, \ldots, n$;

ii. All $\tilde{x}_{i}(t), i=1,2, \ldots, n$ are (ii)-gH differentiable and all $a_{i j} \geqslant 0, i, j=1,2, \ldots, n$.

\section{Defuzzification method}

Defuzzification method is advantageous for a fuzzy problem for two important views, i.e. to obtain the crisp solution and for the researchers who cannot analyze the fuzzy results of the phenomena. Defuzzification is the process of producing a quantifiable result in fuzzy logic, when given the fuzzy sets and the corresponding degree of membership function. There are several defuzzification techniques among them some of the common and useful aids are as follows.

\subsection{Centre of area (COA) method}

In this method defuzzification of a fuzzy number $u$ is carried out by using the following formula

$$
\operatorname{COA}(\mathfrak{u})=\frac{\int_{\sup (\mathfrak{u})} x \Omega_{\mathfrak{u}}(x) d x}{\int_{\sup (\mathfrak{u})} \Omega_{\mathfrak{u}}(x) d x} .
$$

Therefore, to defuzzify a triangular fuzzy number $\Lambda=\left(a_{1}, a_{2}, a_{3}\right)$ we get

$$
\operatorname{COA}(\Lambda)=\frac{\int_{x=a_{1}}^{a_{2}} \times \frac{x-a_{1}}{a_{2}-a_{1}} d x+\int_{x=a_{2}}^{a_{3}} \times \frac{a_{3}-x}{a_{3}-a_{2}} d x}{\int_{x=a_{1}}^{a_{2}} \frac{x-a_{1}}{a_{2}-a_{1}} d x+\int_{x=a_{2}}^{a_{3}} \frac{a_{3}-x}{a_{3}-a_{2}} d x} .
$$

On simplifying we get

$$
\operatorname{COA}(\Lambda)=\frac{\left(a_{1}+a_{2}+a_{3}\right)}{3}
$$




\subsection{Bisector of area $(B O A)$ method}

The $\mathrm{BOA}$ is the vertical line that divides the region into two sub regions of equal area, i.e.,

Area of the left-hand side of the bisector $=$ Area of the right-hand side of the bisector, which in case of a triangular fuzzy number $\Lambda$ is attained as,

$$
\frac{1}{2}\left\{\left(\operatorname{BOA}(\Lambda)-a_{1}\right)+\left(\operatorname{BOA}(\Lambda)-a_{2}\right)\right\} \cdot 1=\frac{1}{2}\left\{\left(a_{3}-\operatorname{BOA}(\Lambda)\right)+\left(a_{2}-\operatorname{BOA}(\Lambda)\right)\right\} \cdot 1 .
$$

On solving we get

$$
\operatorname{BOA}(\Lambda)=\frac{a_{1}+2 a_{2}+a_{3}}{4}
$$

\subsection{Largest of maxima (LOM) method}

The largest of maxima of a fuzzy number $u$, i.e., $\operatorname{LOM}(u)$ takes the largest among all $x \in \sup (u)$ with maximum membership degree as the crisp value. For example, according to LOM, defuzzified value of a triangular fuzzy number $\Lambda$ is

$$
\operatorname{LOM}(\Lambda)=a_{2}
$$

\subsection{Smallest of maxima (SOM) method}

The smallest of maxima of a fuzzy number $u$, i.e. SOM $(u)$ takes the smallest among all $x \in \sup (u)$ with maximum membership degree as the crisp value. So, for triangular fuzzy number $\Lambda$ it is

$$
\operatorname{SOM}(\Lambda)=a_{2}
$$

\subsection{Mean of maxima (MOM) method}

In this method, only active rules with highest degree of fulfillment are taken into account. The output is

$$
\operatorname{MOM}(u)=\frac{1}{2}(\operatorname{LOM}(u)+\operatorname{SOM}(u)) .
$$

Therefore, in case of triangular fuzzy number it is

$$
\operatorname{MOM}(\Lambda)=a_{2} .
$$

\subsection{Regular weighted point (RWP) method}

Let $\Lambda=\left(a_{1}, a_{2}, a_{3}\right)$ be a triangular fuzzy number with $\alpha$-levels $[\Lambda]^{\alpha}=\left[\Lambda_{1}(\alpha), \Lambda_{2}(\alpha)\right]$, then the regular weighted point is given by

$$
\operatorname{RWP}(\Lambda)=\frac{\int_{0}^{1} \frac{\Lambda_{1}(\alpha)+\Lambda_{2}(\alpha)}{2} \xi(\alpha) d \alpha}{\int_{0}^{1} \xi(\alpha) d \alpha}
$$

where

$$
\xi(\alpha)= \begin{cases}1-2 \alpha, & \text { if } \alpha \in\left[0, \frac{1}{2}\right] \\ 2 \alpha-1, & \text { if } \alpha \in\left[\frac{1}{2}, 1\right] .\end{cases}
$$

Thus,

$$
\operatorname{RWP}(\Lambda)=\frac{\int_{0}^{0.5} \frac{\Lambda_{1}(\alpha)+\Lambda_{2}(\alpha)}{2}(1-2 \alpha) d \alpha+\int_{0.5}^{1} \frac{\Lambda_{1}(\alpha)+\Lambda_{2}(\alpha)}{2}(2 \alpha-1) d \alpha}{\int_{0}^{0.5}(1-2 \alpha) d \alpha+\int_{0.5}^{1}(2 \alpha-1) d \alpha}
$$

On manipulation, we have

$$
\operatorname{RWP}(\Lambda)=\frac{a_{1}+2 a_{2}+a_{3}}{4} .
$$


4.7. Graded mean integration value (GMIV) method

Let $\Lambda=\left(a_{1}, a_{2}, a_{3}\right)$ be a triangular fuzzy number with $\alpha$-levels $[\Lambda]^{\alpha}=\left[\Lambda_{1}(\alpha), \Lambda_{2}(\alpha)\right]$, where

$$
\begin{aligned}
\Lambda_{1}(\alpha) & =\min \left\{\Omega_{\Lambda}(x) \geqslant \alpha, \forall \alpha \in[0,1]\right\} \\
& =\min \left\{x: x \in\left[\left(a_{1}+\left(a_{2}-a_{1}\right) \alpha\right),\left(a_{3}-\left(a_{3}-a_{2}\right) \alpha\right)\right]\right\}=a_{1}+\left(a_{2}-a_{1}\right) \alpha
\end{aligned}
$$

and

$$
\begin{aligned}
\Lambda_{2}(\alpha) & =\max \left\{\Omega_{\Lambda}(x) \geqslant \alpha, \forall \alpha \in[0,1]\right\} \\
& =\max \left\{x: x \in\left[\left(a_{1}+\left(a_{2}-a_{1}\right) \alpha\right),\left(a_{3}-\left(a_{3}-a_{2}\right) \alpha\right)\right]\right\}=a_{3}-\left(a_{3}-a_{2}\right) \alpha,
\end{aligned}
$$

then

$$
\operatorname{GMIV}_{\lambda}(\Lambda)=\frac{\int_{\alpha=0}^{1}\left\{(1-\lambda) \Lambda_{1}(\alpha)+\lambda \Lambda_{2}(\alpha)\right\} \alpha \mathrm{d} \alpha}{\int_{\alpha=0}^{1} \alpha \mathrm{d} \alpha},
$$

where $\lambda>0$ is a parameter. After simplification, we obtain

$$
\operatorname{GMIV}_{\lambda=0.5}(\Lambda)=\frac{a_{1}+4 a_{2}+a_{3}}{6} .
$$

\subsection{Centre of approximated interval (COAI) of a fuzzy number}

Let $[a, b]$ be approximated interval of a fuzzy number $u$ and let $\alpha$-level of $u$ be $[u]^{\alpha}=\left[u_{1}(\alpha), u_{2}(\alpha)\right]$. Calculating the distance between fuzzy number $u$ and interval $[a, b]$, for $w \in(0,1]$,

$$
D_{F}(u,[a, b])=\sqrt{\int_{\alpha=0}^{w}\left(u_{1}(\alpha)-a\right)^{2} d \alpha+\int_{\alpha=0}^{w}\left(u_{2}(\alpha)-b\right)^{2} d \alpha .}
$$

Since $[a, b]$ is the nearest interval of $u$, i.e., $\min D_{F}(u,[a, b]), \forall a, b$, therefore, $\frac{\partial D_{F}}{\partial a}=0, \frac{\partial D_{F}}{\partial a}=0$, principal minors of Hessian matrix $H=\left[\begin{array}{ll}\frac{\partial^{2} D_{F}}{\partial a^{2}} & \frac{\partial^{2} D_{F}}{\partial a \partial b} \\ \frac{\partial^{2} D_{F}}{\partial b \partial a} & \frac{\partial^{2} D_{F}}{\partial b^{2}}\end{array}\right]$ are positive, i.e., $\Delta_{1}=\frac{\partial^{2} D_{F}}{\partial a^{2}}>0$ and $\Delta_{2}=$ $\left[\begin{array}{ll}\frac{\partial^{2} D_{F}}{\partial a^{2}} & \frac{\partial^{2} D_{F}}{\partial a \partial b} \\ \frac{\partial^{2} D_{F}}{\partial b \partial a} & \frac{\partial^{2} D_{F}}{\partial b^{2}}\end{array}\right]>0$. Here, $\frac{\partial D_{F}}{\partial a}=\int_{\alpha=0}^{w} 2\left(A_{1}(\alpha)-a\right)(-1) d \alpha$, which implies $a=\frac{1}{w} \int_{\alpha=0}^{w} A_{1}(\alpha) d \alpha$ and $\frac{\partial D_{F}}{\partial b}=\int_{\alpha=0}^{w} 2\left(A_{2}(\alpha)-b\right)(-1) d \alpha$ implies $b=\frac{1}{w} \int_{\alpha=0}^{w} A_{2}(\alpha) d \alpha$. Moreover, $\frac{\partial^{2} D_{F}}{\partial a^{2}}=2 \int_{\alpha=0}^{w} d \alpha=2 w$, $\frac{\partial^{2} D_{F}}{\partial a \partial b}=\frac{\partial^{2} D_{F}}{\partial b \partial a}=0, \frac{\partial^{2} D_{F}}{\partial b^{2}}=2 \int_{\alpha=0}^{w} d \alpha=2 w$. Therefore, the Hessian matrix becomes $\mathrm{H}=\left[\begin{array}{cc}2 w & 0 \\ 0 & 2 w\end{array}\right]$ with $\Delta_{1}=2 w>0$ and $\Delta_{2}=\left|\begin{array}{cc}2 w & 0 \\ 0 & 2 w\end{array}\right|=4>0$. Hence

$$
\operatorname{COAI}(\Lambda)=\frac{a+b}{2}=\frac{1}{w} \int_{\alpha=0}^{w} \frac{u_{1}(\alpha)+u_{2}(\alpha)}{2} \mathrm{~d} \alpha .
$$

Thus, for a triangular fuzzy number $\Lambda=\left(a_{1}, a_{2}, a_{3}\right)$, the defuzzified form, using COAI method is

$$
\operatorname{COAI}(\Lambda)=\frac{a_{1}+2 a_{2}+a_{3}}{4}
$$

\section{Arms race model}

An interesting application that leads to a system of differential equations is the study of arms race. When there is a dispute between two nations, a war arises between the nations so that the nations are then said to be in conflict. In this case, each nation will try to acquire arms to defend itself against a possible attack by the opponent nation in a conflict. This begins the race for accumulation of arms. 
Here, we will present a model for arms race, which is known as Richardson's model. In this model following assumptions are taken into account.

(i) The rate of increase of armament expenditure is proportional to the other countries expenditure.

(ii) The rate of decrease of armament expenditure is proportional to its own expenditure.

(iii) The rate of change of arms expenditure for a country has a constant component that measures the level of antagonism of that country towards the other.

(iv) The effects of all these assumptions are additive.

Let $x(t)$ and $y(t)$ denote the expenditure incurred by two countries on armament at a time $t$, then under the assumptions (i)-(iv), the system of differential equation is structured as

$$
\frac{d x(t)}{d t}=a y(t)-p x(t)+r, \quad \frac{d y(t)}{d t}=b x(t)-q y(t)+s,
$$

where, $p$ and $q$ represent the costs of the equipment, $r$ and $s$ are the leftovers of the countries. On the other hand, $a$ and $b$ illustrate the first assumption. All the parameters are taken to be positive constants, whereas $r$ and $s$ can take any value. Positive values arise if the countries have internal attitudes of distrust of each other. In matrix notation Eq. (5.1) may be written as

$$
\dot{X}=A X+B, \quad X(0)=\left(\begin{array}{l}
x(0) \\
y(0)
\end{array}\right)
$$

where

$$
\dot{X}(0)=\left(\begin{array}{l}
\dot{x}(t) \\
\dot{y}(t)
\end{array}\right), X(t)=\left(\begin{array}{l}
x(t) \\
y(t)
\end{array}\right), A=\left(\begin{array}{cc}
-p & a \\
b & -q
\end{array}\right), A=\left(\begin{array}{l}
r \\
s
\end{array}\right) .
$$

The nature of the solutions of the system will depend upon the eigenvalues of the matrix $A$, i.e., on the roots of the following characteristic equation

$$
\left|\begin{array}{cc}
-p-\kappa & a \\
b & -q-\kappa
\end{array}\right|=k^{2}+(p+q) \kappa+(p q-a b)=0 .
$$

So, the roots are obtained as

$$
\frac{-(p+q) \pm \sqrt{(p-q)^{2}+4 a b}}{2} .
$$

As $a$ and $b$ are positive, so the eigenvalues are real and distinct. For $p>0$ and $q>0$, it follows that the roots are negative if

$$
\frac{-(p+q) \pm \sqrt{(p-q)^{2}+4 a b}}{2}<0,
$$

which results $(p q-a b)>0$, while the roots will have opposite signs, if $(p q-a b)<0$. The presence of a positive eigenvalue is disturbing, since it will lead to an exponential function that becomes unbounded as time increases and the situation that may result in a runaway arms race.

\section{Applications}

Consider the following ARM having positive parameters

$$
\frac{\mathrm{dX}(\mathrm{t})}{\mathrm{dt}}=\left(\begin{array}{cc}
-3 & 2 \\
3 & -4
\end{array}\right) \times(\mathrm{t})+\left(\begin{array}{l}
1 \\
2
\end{array}\right)
$$

with fuzzy initial condition $X(0)=\left(\begin{array}{l}\widetilde{\gamma}_{1} \\ \widetilde{\gamma}_{2}\end{array}\right)$, where $\widetilde{\gamma}_{1}=\widetilde{\gamma}_{2}=(70,100,130)$ and $X(t)=\left(\begin{array}{l}x(t) \\ y(t)\end{array}\right)$. 


\subsection{Zadeh's extension principle}

Let the nonhomogeneous system (6.1) has a solution of the form

$$
X_{p}(t)=\left(\begin{array}{l}
\mu \\
\eta
\end{array}\right)
$$

Substituting Eq. (6.2) in the system (6.1) we have

$$
\left(\begin{array}{l}
0 \\
0
\end{array}\right)=\left(\begin{array}{cc}
-3 & 2 \\
3 & -4
\end{array}\right)\left(\begin{array}{l}
\mu \\
\eta
\end{array}\right)+\left(\begin{array}{l}
1 \\
2
\end{array}\right),
$$

which on simplifying gives

$$
X_{p}(t)=\left(\begin{array}{l}
\mu \\
\eta
\end{array}\right)=\left(\begin{array}{l}
\frac{4}{3} \\
\frac{3}{2}
\end{array}\right)
$$

Thus, the general solution of Eq. (6.1) is attained as

$$
X(t)=\left(\begin{array}{cc}
2 e^{-6 t} & e^{-t} \\
-3 e^{-6 t} & e^{-t}
\end{array}\right)\left(\begin{array}{l}
c_{1} \\
c_{2}
\end{array}\right)+\left(\begin{array}{l}
\mu \\
\eta
\end{array}\right)=\left(\begin{array}{c}
\frac{4}{3} \\
\frac{3}{2}
\end{array}\right)
$$

Using the initial conditions and calculating values of $c_{1}$ and $c_{2}$ we get the solution

$$
X(t)=\left(\begin{array}{cc}
\frac{2 e^{-6 t}+3 e^{-t}}{5} & \frac{2}{5}\left(-e^{-6 t}+3 e^{-t}\right) \\
\frac{-3}{5}\left(e^{-6 t}-3 e^{-t}\right) & \frac{3 e^{-6 t}+2 e^{-t}}{5}
\end{array}\right)\left(\begin{array}{l}
\widetilde{\gamma}_{1} \\
\widetilde{\gamma}_{2}
\end{array}\right)+\left(\begin{array}{c}
\frac{e^{-6 t}}{15}-\frac{7 e^{-t}}{5}+\frac{4}{3} \\
-\frac{e^{-6 t}}{10}-\frac{7 e^{-t}}{5}+\frac{3}{2}
\end{array}\right) .
$$

Since, $\frac{2 e^{-6 t}+3 e^{-t}}{5}>0, \frac{2}{5}\left(-e^{-6 t}+3 e^{-t}\right)>0$, and $\frac{-3}{5}\left(e^{-6 t}-3 e^{-t}\right)>0, \frac{3 e^{-6 t}+2 e^{-t}}{5}>0$, therefore, Eq. (6.3) can be written in expanded form as

$$
\begin{aligned}
& x(t)=\frac{2 e^{-6 t}+3 e^{-t}}{5} \widetilde{\gamma}_{1}+\frac{2}{5}\left(-e^{-6 t}+3 e^{-t}\right) \widetilde{\gamma}_{2}+\frac{e^{-6 t}}{15}-\frac{7 e^{-t}}{5}+\frac{4}{3} \\
& y(t)=\frac{-3}{5}\left(e^{-6 t}-3 e^{-t}\right) \widetilde{\gamma}_{1}+\frac{3 e^{-6 t}+2 e^{-t}}{5} \widetilde{\gamma}_{2}-\frac{e^{-6 t}}{10}-\frac{7 e^{-t}}{5}+\frac{3}{2} .
\end{aligned}
$$

Substituting $\alpha$-levels of $\widetilde{\gamma}_{1}$ and $\widetilde{\gamma}_{2}$ we get

$$
\begin{aligned}
& x_{1}(t ; \alpha)=(70+30 \alpha) e^{-t}+\frac{1}{15} e^{-6 t}-\frac{7}{5} e^{-t}+\frac{4}{3}, x_{2}(t ; \alpha)=(130-30 \alpha) e^{-t}+\frac{1}{15} e^{-6 t}-\frac{7}{5} e^{-t}+\frac{4}{3} \\
& y_{1}(t ; \alpha)=(70+30 \alpha) e^{-t}+\frac{1}{10} e^{-6 t}-\frac{7}{5} e^{-t}+\frac{3}{2}, \quad y_{2}(t ; \alpha)=(130-30 \alpha) e^{-t}+\frac{1}{10} e^{-6 t}-\frac{7}{5} e^{-t}+\frac{3}{2} .
\end{aligned}
$$

\subsubsection{Defuzzification of the solution}

To defuzzify the obtained solutions (6.4) of system (6.1), the scheme is proceeded as follows. Calculating Eqs. (6.4) at a particular time, we get

$$
x_{1}\left(\frac{1}{4} ; \alpha\right)=54.7739424+23.36402349 \alpha, \quad x_{2}\left(\frac{1}{4} ; \alpha\right)=101.5019894-23.36402349 \alpha,
$$


and

$$
y_{1}\left(\frac{1}{4} ; \alpha\right)=54.9034207+23.36402349 \alpha, \quad y_{2}\left(\frac{1}{4} ; \alpha\right)=101.6314677-23.36402349 \alpha
$$

Taking the mean of the respective lower and upper functions, we attain

$$
\frac{x_{1}\left(\frac{1}{4} ; \alpha\right)+x_{2}\left(\frac{1}{4} ; \alpha\right)}{2}=78.1379659=M_{1}, \frac{y_{1}\left(\frac{1}{4} ; \alpha\right)+y_{2}\left(\frac{1}{4} ; \alpha\right)}{2}=78.2674442=M_{2} .
$$

In addition, on using Eq. (4.1), defuzzified solution of Eq. (6.1) is also obtained as

$$
\begin{aligned}
& \operatorname{RWP}(\widetilde{x})=\frac{\int_{0}^{1} M_{1} \xi(\alpha) d \alpha}{\int_{0}^{1} \xi(\alpha) d \alpha}=78.1379659=\operatorname{GMIV}_{0.5}(\widetilde{x})=M_{1}, \\
& \operatorname{RWP}(\widetilde{y})=\frac{\int_{0}^{1} M_{2} \xi(\alpha) d \alpha}{\int_{0}^{1} \xi(\alpha) d \alpha}=78.2674442=\operatorname{GMIV}_{0.5}(\widetilde{y})=M_{2} .
\end{aligned}
$$

Hence, the defuzzified solutions of system (6.1) are $x=78.138$ and $y=78.267$.

\subsection{Generalized H-derivative approach}

For the cases of gH-differentiability, let $x(t)$ and $y(t)$ in system (6.1) be fuzzy-valued functions, then the governing problem will undergo the following cases.

\subsubsection{When $x(t)$ and $y(t)$ are (i)-gH differentiable}

In this case, system (6.1) can be expressed in its $\alpha$-level sets as

$$
\begin{aligned}
& \dot{x}_{1}(t ; \alpha)=0 x_{1}(t ; \alpha)-3 x_{2}(t ; \alpha)+2 y_{1}(t ; \alpha)+0 y_{2}(t ; \alpha)+1, \\
& \dot{x}_{2}(t ; \alpha)=-3 x_{1}(t ; \alpha)+0 x_{2}(t ; \alpha)+0 y_{1}(t ; \alpha)+2 y_{2}(t ; \alpha)+1, \\
& \dot{y}_{1}(t ; \alpha)=-3 x_{1}(t ; \alpha)+0 x_{2}(t ; \alpha)+0 y_{1}(t ; \alpha)-4 y_{2}(t ; \alpha)+2, \\
& \dot{y}_{2}(t ; \alpha)=0 x_{1}(t ; \alpha)+3 x_{2}(t ; \alpha)-4 y_{1}(t ; \alpha)+0 y_{2}(t ; \alpha)+2,
\end{aligned}
$$

with fuzzy initial conditions

$$
\left(\begin{array}{l}
x_{1}(0 ; \alpha) \\
x_{2}(0 ; \alpha) \\
y_{1}(0 ; \alpha) \\
y_{2}(0 ; \alpha)
\end{array}\right)=\left(\begin{array}{c}
70+30 \alpha \\
130-30 \alpha \\
70+30 \alpha \\
130-30 \alpha
\end{array}\right)
$$

Solving the above system, we get the fuzzy solutions as

$$
\begin{aligned}
& x_{1}(t ; \alpha)=-6(1-\alpha) e^{t}-24(1-\alpha) e^{6 t}+\frac{493}{5} e^{-t}+\frac{1}{15} e^{-6 t}+\frac{4}{3} \\
& x_{2}(t ; \alpha)=6(1-\alpha) e^{t}+24(1-\alpha) e^{6 t}+\frac{493}{5} e^{-t}+\frac{1}{15} e^{-6 t}+\frac{4}{3} \\
& y_{1}(t ; \alpha)=6(1-\alpha) e^{t}-36(1-\alpha) e^{6 t}+\frac{493}{5} e^{-t}-\frac{1}{10} e^{-6 t}+\frac{3}{2} \\
& y_{2}(t ; \alpha)=-6(1-\alpha) e^{t}+36(1-\alpha) e^{6 t}+\frac{493}{5} e^{-t}-\frac{1}{10} e^{-6 t}+\frac{3}{2} .
\end{aligned}
$$

Calculating the crisp values of Eqs. (6.6) at $\alpha=0,1$ and $t=\frac{1}{4}$ we get

$$
x_{1}\left(\frac{1}{4} ; 0\right)=-37.127, \quad x_{2}\left(\frac{1}{4} ; 0\right)=193.403, \quad x_{1}\left(\frac{1}{4} ; 1\right)=x_{2}\left(\frac{1}{4} ; 1\right)=78.138
$$


Hence

$$
x=(-37.127,78.138,193.403) .
$$

Similarly

$$
y_{1}\left(\frac{1}{4} ; 0\right)=-75.369, y_{2}\left(\frac{1}{4} ; 0\right)=231.904, y_{1}\left(\frac{1}{4} ; 1\right)=y_{2}\left(\frac{1}{4} ; 1\right)=78.267
$$

Therefore

$$
y=(-75.369,78.267,231.904) .
$$

The defuzzified solutions of Eqs. (6.6) obtained by using Eqs. (6.7)-(6.8) and the techniques defined in Section 4 are $x=78.138$ and $y=78.267$.

\subsubsection{When $\mathrm{x}(\mathrm{t})$ is (i)-gH differentiable and $\mathrm{y}(\mathrm{t})$ is (ii)-gH differentiable}

Converting Eq. (6.1) into $\alpha$-level sets under this case, we get

$$
\begin{aligned}
& \dot{x}_{1}(t ; \alpha)=0 x_{1}(t ; \alpha)-3 x_{2}(t ; \alpha)+0 y_{1}(t ; \alpha)+2 y_{2}(t ; \alpha)+1, \\
& \dot{x}_{2}(t ; \alpha)=-3 x_{1}(t ; \alpha)+0 x_{2}(t ; \alpha)+2 y_{1}(t ; \alpha)+0 y_{2}(t ; \alpha)+1, \\
& \dot{y}_{1}(t ; \alpha)=-3 x_{1}(t ; \alpha)+0 x_{2}(t ; \alpha)-4 y_{1}(t ; \alpha)+0 y_{2}(t ; \alpha)+2, \\
& \dot{y}_{2}(t ; \alpha)=0 x_{1}(t ; \alpha)+3 x_{2}(t ; \alpha)+0 y_{1}(t ; \alpha)-4 y_{2}(t ; \alpha)+2,
\end{aligned}
$$

with the initial conditions as defined in Eq. (6.5). The solution is attained as

$$
\begin{aligned}
& x_{1}(t ; \alpha)=-6(1-\alpha) e^{-3 t}-24(1-\alpha) e^{2 t}+\frac{493}{5} e^{-t}+\frac{1}{15} e^{-6 t}+\frac{4}{3} \\
& x_{2}(t ; \alpha)=6(1-\alpha) e^{-3 t}+24(1-\alpha) e^{2 t}+\frac{493}{5} e^{-t}+\frac{1}{15} e^{-6 t}+\frac{4}{3}, \\
& y_{1}(t ; \alpha)=-18(1-\alpha) e^{-3 t}-12(1-\alpha) e^{2 t}+\frac{493}{5} e^{-t}-\frac{1}{10} e^{-6 t}+\frac{3}{2}, \\
& y_{2}(t ; \alpha)=18(1-\alpha) e^{-3 t}+12(1-\alpha) e^{2 t}+\frac{493}{5} e^{-t}-\frac{1}{10} e^{-6 t}+\frac{3}{2} .
\end{aligned}
$$

Calculating the crisp values of Eqs. (6.9) at $\alpha=0,1$ and $t=\frac{1}{4}$ we get

$$
\begin{aligned}
& x_{1}\left(\frac{1}{4} ; 0\right)=35.734, \quad x_{2}\left(\frac{1}{4} ; 0\right)=120.541, \quad x_{1}\left(\frac{1}{4} ; 1\right)=x_{2}\left(\frac{1}{4} ; 1\right)=78.138 \\
& y_{1}\left(\frac{1}{4} ; 0\right)=49.980, \quad y_{2}\left(\frac{1}{4} ; 0\right)=106.555, \quad y_{1}\left(\frac{1}{4} ; 1\right)=y_{2}\left(\frac{1}{4} ; 1\right)=78.267
\end{aligned}
$$

So,

$$
x=(35.734,78.138,120.541), y=(49.980,78.267,106.555) .
$$

Its defuzzified solutions, using the aforementioned techniques in Section 4 , are $x=78.138$ and $y=78.267$.

\subsubsection{When $\mathrm{x}(\mathrm{t})$ is (ii)-gH differentiable and $\mathrm{y}(\mathrm{t})$ is $(\mathrm{i})-\mathrm{gH}$ differentiable}

Expressing Eq. (6.1) in its $\alpha$-level sets under this case we get

$$
\begin{aligned}
& \dot{x}_{1}(t ; \alpha)=-3 x_{1}(t ; \alpha)+0 x_{2}(t ; \alpha)+2 y_{1}(t ; \alpha)+0 y_{2}(t ; \alpha)+1, \\
& \dot{x}_{2}(t ; \alpha)=0 x_{1}(t ; \alpha)-3 x_{2}(t ; \alpha)+2 y_{1}(t ; \alpha)+0 y_{2}(t ; \alpha)+1, \\
& \dot{y}_{1}(t ; \alpha)=0 x_{1}(t ; \alpha)+3 x_{2}(t ; \alpha)+0 y_{1}(t ; \alpha)-4 y_{2}(t ; \alpha)+2, \\
& \dot{y}_{2}(t ; \alpha)=3 x_{1}(t ; \alpha)+0 x_{2}(t ; \alpha)-4 y_{1}(t ; \alpha)+0 y_{2}(t ; \alpha)+2,
\end{aligned}
$$


with the initial conditions defined in Eq. (6.5). After some manipulation, we get the solutions as

$$
\begin{aligned}
& x_{1}(t ; \alpha)=-6(1-\alpha) e^{3 t}-24(1-\alpha) e^{-2 t}+\frac{493}{5} e^{-t}+\frac{1}{15} e^{-6 t}+\frac{4}{3} \\
& x_{2}(t ; \alpha)=6(1-\alpha) e^{3 t}+24(1-\alpha) e^{-2 t}+\frac{493}{5} e^{-t}+\frac{1}{15} e^{-6 t}+\frac{4}{3} \\
& y_{1}(t ; \alpha)=-18(1-\alpha) e^{3 t}-12(1-\alpha) e^{-2 t}+\frac{493}{5} e^{-t}-\frac{1}{10} e^{-6 t}+\frac{3}{2} \\
& y_{2}(t ; \alpha)=18(1-\alpha) e^{3 t}+12(1-\alpha) e^{-2 t}+\frac{493}{5} e^{-t}-\frac{1}{10} e^{-6 t}+\frac{3}{2} .
\end{aligned}
$$

Calculating the crisp values of Eqs. (6.10) at $\alpha=0,1$ and $t=\frac{1}{4}$ we get

$$
\begin{aligned}
& x_{1}\left(\frac{1}{4} ; 0\right)=50.879, \quad x_{2}\left(\frac{1}{4} ; 0\right)=105.397, \quad x_{1}\left(\frac{1}{4} ; 1\right)=x_{2}\left(\frac{1}{4} ; 1\right)=78.138 \\
& y_{1}\left(\frac{1}{4} ; 0\right)=32.883, \quad y_{2}\left(\frac{1}{4} ; 0\right)=123.652, \quad y_{1}\left(\frac{1}{4} ; 1\right)=y_{2}\left(\frac{1}{4} ; 1\right)=78.267
\end{aligned}
$$

Thus

$$
x=(50.879,78.138,105.397), y=(32.883,78.267,123.652) .
$$

The defuzzified solutions of Eqs. (6.10) obtained by using Eq. (6.11) and the techniques, defined in Section 4 , are $x=78.138$ and $y=78.267$.

\subsubsection{When $\mathrm{x}(\mathrm{t})$ and $\mathrm{y}(\mathrm{t})$ are (ii)-gH differentiable}

In this case, system (6.1) can be expressed in its $\alpha$-level sets as

$$
\begin{aligned}
& \dot{x}_{1}(t ; \alpha)=-3 x_{1}(t ; \alpha)+0 x_{2}(t ; \alpha)+0 y_{1}(t ; \alpha)+2 y_{2}(t ; \alpha), \\
& \dot{x}_{2}(t ; \alpha)=0 x_{1}(t ; \alpha)-32(t ; \alpha)+2 y_{1}(t ; \alpha)+0 y_{2}(t ; \alpha), \\
& \dot{y}_{1}(t ; \alpha)=0 x_{1}(t ; \alpha)+3 x_{2}(t ; \alpha)-4 y_{1}(t ; \alpha)+0 y_{2}(t ; \alpha), \\
& \dot{y}_{2}(t ; \alpha)=3 x_{1}(t ; \alpha)+0 x_{2}(t ; \alpha)+0 y_{1}(t ; \alpha)-4 y_{2}(t ; \alpha),
\end{aligned}
$$

with the initial conditions defined in Eq. (6.5). After some manipulation, we get the solution as

$$
\begin{aligned}
& x_{1}(t ; \alpha)=\left(\frac{463}{5}+6 \alpha\right) e^{-t}-\left(\frac{359}{15}-24 \alpha\right) e^{-6 t}+\frac{4}{3} \\
& x_{2}(t ; \alpha)=\left(\frac{523}{5}-6 \alpha\right) e^{-t}+\left(\frac{361}{15}-24 \alpha\right) e^{-6 t}+\frac{4}{3} \\
& y_{1}(t ; \alpha)=\left(\frac{523}{5}-6 \alpha\right) e^{-t}+\left(\frac{361}{15}-36 \alpha\right) e^{-6 t}+\frac{3}{2} \\
& y_{2}(t ; \alpha)=\left(\frac{463}{5}+6 \alpha\right) e^{-t}+\left(\frac{359}{15}-36 \alpha\right) e^{-6 t}+\frac{3}{2} .
\end{aligned}
$$

Calculating the crisp values of Eqs. (6.12) at $\alpha=0,1$ and $t=\frac{1}{4}$ we get

$$
\begin{aligned}
& x_{1}\left(\frac{1}{4} ; 0\right)=68.11, \quad x_{2}\left(\frac{1}{4} ; 0\right)=88.166, y_{1}\left(\frac{1}{4} ; 0\right)=74.908, \quad y_{2}\left(\frac{1}{4} ; 0\right)=81.627 \\
& x_{1}\left(\frac{1}{4} ; 1\right)=x_{2}\left(\frac{1}{4} ; 1\right)=78.138, \quad y_{1}\left(\frac{1}{4} ; 1\right)=y_{2}\left(\frac{1}{4} ; 1\right)=78.267 .
\end{aligned}
$$

So,

$$
x=(68.11,78.138,88.166), y=(74.908,78.267,81.627) .
$$

Hence, defuzzified solutions of Eqs. (6.12) obtained by using Eq. (6.13) and the aforementioned techniques in Section 4 , are $x=78.138$ and $y=78.267$. 


\section{Conclusion}

In this paper, system of fuzzy differential equations was taken into account to construct and estimate uncertain parameters of an arms race model, which is considered to be an important area of research in international military planning. Two different approaches, namely, Zadeh's extension principle and generalized Hukuhara derivative concept, were applied to elucidate the fuzzy solutions of the governing model. We also discussed different defuzzification methods to defuzzify the fuzzy solutions and attain its crisp solutions. The analytical solutions acquired in fuzzy sense, are beneficial to locate all the expenditures that is suitable for the countries to incur on armament at any time period with a fixed equipment costs and leftover. The likelihoods of expenditures on armament are achieved in the form of lower and upper levels of closed intervals for each time period. While the defuzzified solution shows exactly one choice of expenditure for each country at a certain time. Comprehensively, the whole deliberation reaches its conclusion with the following remarks.

- Demonstrating ARM with fuzzy numbers enabled to meet the imprecise parameters as well, which is approvingly advantageous for the military planners to analyze the armament expenditures in a more precise manner.

- Zadeh's extension principle and gH-derivative approach, having significant place in fuzzy calculus, efficiently made it possible to obtain the fuzzy solutions of the governing model in both the cases, structured in crisp form or in fuzzy sense, accordingly.

- Most of the time in real-world applications, results are necessitated as single terms but with the consideration of all uncertainties. Defuzzification techniques are surely very helpful for any researcher to have a specific result after defuzzifying the fuzzy solutions.

Hence, this endeavor provides an aid to interpret the SFDEs in two ways, i.e. in crisp and in fuzzy sense. In future, we seek to apply these concepts to different types of differential equations in fuzzy environments.

\section{References}

[1] T. Allahviranloo, Z. Gouyandeh, A. Armand, A. Hasanoglu, On fuzzy solutions for heat equation based on generalized Hukuhara differentiability, Fuzzy Sets and Systems, 265 (2015), 1-23. 1, 2.3

[2] L. C. Barros, R. C. Bassanezi, P. A. Tonelli, Fuzzy modelling in population dynamics, Ecol. Model., 128 (2000), 27-33. 1

[3] B. Bede, I. J. Rudas, J. Fodor, Friction model by using fuzzy differential equations, Lecture Notes in Comput. Sci., 4529 (2007), 23-32. 1

[4] B. Bede, L. Stefanini, Generalized differentiability of fuzzy-valued functions, Fuzzy Sets and Systems, 230 (2013), 119141. 1, 2.1, 2.3

[5] A. Bencsik, B. Bede, J. Tar, J. Fodor, Fuzzy differential equations in modeling hydraulic differential servo cylinders, Third Romanian-Hungarian joint symposium on applied computational intelligence (SACI), Timisoara, Romania, (2006). 1

[6] J. J. Buckley, T. Feuring, Fuzzy differential equations, Fuzzy Sets and Systems, 110 (2000), 43-54. 1

[7] J. Casasnovas, F. Rosselló, Averaging fuzzy biopolymers, Fuzzy Sets and Systems, 152 (2005), 139-158. 1

[8] S. H. Chen, S. T. Wang, S. M. Chang, Some properties of graded mean integration representation of L-R type fuzzy numbers, Tamsui Oxf. J. Math. Sci., 22 (2006), 185-208. 1

[9] G. L. Diniz, J. F. R. Fernandes, J. F. C. A. Meyer, L. C. Barros, A fuzzy Cauchy problem modelling the decay of the biochemical oxygen demand in water, Proceedings Joint 9th IFSA World Congress and 20th NAFIPS International Conference, Vancouver, BC, Canada, 1 (2001), 512-516. 1

[10] M. S. El Naschie, From experimental quantum optics to quantum gravity via a fuzzy Kähler manifold, Chaos Solitons Fractals, 25 (2005), 969-977. 1

[11] O. S. Fard, An iterative scheme for the solution of generalized system of linear fuzzy differential equations, World Appl. Sci. J., 7 (2009), 1597-1604. 1

[12] N. Gasilov, S.. E. Amrahov, A. G. Fatullayev, A geometric approach to solve fuzzy linear systems of differential equations, Appl. Math. Inf. Sci., 5 (2011), 484-499. 1 
[13] W. W. Hill, Several sequential augmentations of Richardson's arms race model, Math. Comput. Model., 16 (1992), 201212. 1

[14] E. Hüllermeier, An approach to modelling and simulation of uncertain dynamical systems, Internat. J. Uncertain. Fuzziness Knowledge-Based Systems, 5 (1997), 117-137. 1

[15] N. A. Khan, O. A. Razzaq, A. Ara, F. Riaz, Numerical solution of system of fractional differential equations in imprecise environment, Numerical Simulation-From Brain Imaging to Turbulent Flow, InTech, (2016) 1

[16] N. A. Khan, O. A. Razzaq, M. Ayyaz, On the solution of fuzzy differential equations by Fuzzy Sumudu Transform, Nonlinear Eng., 4 (2015), 49-60. 1, 2.1

[17] A. Khastan, I. Perfilieva, Z. Alijani, A new fuzzy approximation method to Cauchy problems by fuzzy transform, Fuzzy Sets and Systems, 288 (2016), 75-95. 1

[18] M. J. McLean, An introduction to mathematical models in the social and life sciences, M. Olinick Addison-Wesley Inc., London, (1978), Appl. Math. Modell., 3 (1979), 238-239. 1

[19] M. T. Mizukoshi, L. C. Barros, Y. Chalco-Cano, H. Román-Flores, R. C. Bassanezi, Fuzzy differential equations and the extension principle, Inform. Sci., 177 (2007), 3627-3635. 1

[20] S. P. Mondal, T. K. Roy, First order linear non homogeneous ordinary differential equation in fuzzy environment, Math. Theory Model., 3 (2013), 85-95. 1

[21] S. Naaz, A. Alam, R. Biswas, Effect of different defuzzification methods in a fuzzy based load balancing application, Int. J. Comput. Sci., 8 (2011), 261-267. 1

[22] R. Saneifard, Another method for defuzzification based on regular weighted point, Int. J. Ind. Math., 4 (2011), 147-152.

[23] R. Saneifard, R. Saneifard, A method for defuzzification based on centroid point, Turkish J. Fuzzy Sys., 2 (2011), 36-44. 1

[24] H. Zarei, A. V. Kamyad, A. A. Heydari, Fuzzy modeling and control of HIV infection, Comput. Math. Methods Med., 2012 (2012), 17 pages. 1

[25] Y. Zhang, J.-H. He, S.-Q. Wang, P. Wang, A dye removal model with a fuzzy initial condition, Therm. Sci., 20 (2016), 867-870. 1 